\title{
Self-expanding metallic stent as a bridge to surgery versus emergency surgery for acute obstructive colorectal cancer: a retrospective study
}

This article was published in the following Dove Press journal: Cancer Management and Research

\author{
Yuliuming Wang \\ Hanqing $\mathrm{Hu}$ \\ Meng Wang \\ Xiao Han \\ Qian Zhang \\ Lei Yu \\ Yinggang Chen \\ Guiyu Wang
}

Department of Colorectal Surgery, the Second Affiliated Hospital of Harbin Medical University, Harbin, People's Republic of China
Correspondence: Guiyu Wang Department of Colorectal Surgery, the Second Affiliated Hospital of Harbin Medical University, I57 Baojian Road, Harbin, Heilongjiang province, People's Republic of China Tel +8645I 86296599 Email guiywang@163.com
Purpose: Acute obstructive colorectal cancer is a common emergency that requires decompression immediately. The aim of the study was to compare short-term and long-term results of acute obstructive colorectal cancer treated by the self-expanding metallic stent (SEMS) as a bridge to surgery (BTS) versus emergency surgery.

Patients and methods: We retrospectively reviewed 78 patients who were diagnosed as acute obstructive colorectal cancer that underwent elective surgery after stent insertion (stent group, $\mathrm{N}=37$ ) or emergency surgery (emergency group, $\mathrm{N}=41$ ) from January 2013 to October 2016. The Kaplan-Meier method was conducted to calculate overall survival. Univariate analyses were performed using the Mann-Whitney U analysis, Pearson's chi-square test, and Fisher's exact test. In addition, continuous variables were compared using the Student's $t$-test.

Results: The baseline characteristics were not significantly different between the two groups. The stent group had increased preoperative serum albumin level and decreased ASA risk score. In addition, operation time, diet time, harvested lymph nodes and total stoma creation of the stent group were better than that of the emergency group. The complications and mortality during hospitalization were not significantly different between the two groups. The overall survival was not significantly different while the quality of life of survival patients in the stent group was better than that of the emergency group.

Conclusion: For acute obstructive colorectal cancer, a stent as a BTS seems to be a safe and feasible alternative option for emergency surgery in the management of acute obstructive colorectal cancer.

Keywords: obstructive colorectal cancer, self-expanding metallic stent, bridge to surgery

\section{Introduction}

Colorectal cancer (CRC) is one of the most common cancers in the world. ${ }^{1}$ In China, $\mathrm{CRC}$ is the third and fourth most common cancer in urban and rural districts, respectively. Acute obstructive colorectal cancer, reported to account for about $7-10 \%$ of all colon cancers, is a common emergency that requires decompression to prevent perforation, bacterial translocation, and ischemia. ${ }^{2-4}$ The conventional measure was emergency surgery and usually followed by colostomy. However, it was associated with high morbidity and mortality according to the poor preoperative general condition. ${ }^{5-7}$

In recent years, the self-expanding metallic stent (SEMS) has been widely applied as a palliative treatment measure for malignant colonic obstruction in patients with incurable disease. ${ }^{8}$ SEMS has also been used as a bridge to surgery (BTS) to allow a single-stage surgical procedure later and SEMS seems to be simple and safe in 
comparison with emergency surgery. ${ }^{9-11}$ SEMS as a BTS gives the physicians the opportunity to perform medical resuscitation, optimization of comorbid disorders, bowel preparation, and preoperative tumor staging., ${ }^{4,9}$ However, the complications of SEMS and the potential risk of dissemination have limited the use of SEMS. ${ }^{12-14}$ In addition, the oncologic outcomes and long-term results of elective surgery after stenting versus emergency surgery remain undefined and the published data from the Chinese populations are still lacking.

Accordingly, the aim of the study was to compare preoperative condition, postoperative outcomes and longterm results of acute obstructive colorectal cancer treated by SEMS as a BTS versus emergency surgery.

\section{Patients and methods Medical decision}

Since September 2012, the Endoscopy Unit of the Second Affiliated Hospital of Harbin Medical University has used colonic stenting as a treatment option for patients presenting colorectal cancer with obstructive symptoms. In our hospital, acute obstructive colorectal cancer is diagnosed based on medical history, physical examination, abdominal computed tomography (CT) scan and colonoscopy. Once a patient was diagnosed with acute obstructive colorectal cancer and sent to the Colorectal Surgery Department through an emergency approach, our multidisciplinary treatment (MDT) team would assess the patient's condition. The team, all experienced physicians, consists of two surgeons, an endoscopist and an anesthesiologist. Characteristics including general condition, feasibility for stent insertion, risk of cecal perforation, the American Society of Anesthesiologists Physical Status Classification System (ASA) risk score, results of abdominal and pectoral CT scan and physical examination are used to assess the patient's condition and physicians should attempt to balance the risk of emergency surgery against stent insertion. After the assessment, the team explains the two treatment options (stent insertion or emergency surgery) and the risk of each option to the patient. The patient makes the final decision according to the physicians' suggestions. Emergency surgery is always performed within hours of the diagnosis while the stent procedure is always performed within $24 \mathrm{~h}$ after diagnosis.

\section{Patient selection}

In this study, 84 patients who were diagnosed as acute obstructive colorectal cancer from January 2013 to
October 2016 in our department were retrospectively studied. All the cases were consecutive cases and these cases were all perforation-free cases. In total, 78 patients who had undergone one-stage resection of the primary site were enrolled into this study according to the inclusion and exclusion criteria. The inclusion criteria were: 1) patients diagnosed as colon cancer associated with acute colonic obstruction; 2) patients could not tolerate any oral intake and required a procedure for emergent decompression; and 3) patients underwent one-stage total resection of the primary site through elective surgery or emergency surgery. The exclusion criteria were: 1) patients underwent palliative colostomy without one-stage primary tumor resection during emergency operation; and 2) patients underwent palliative colostomy after stent insertion without one-stage primary tumor resection or only underwent stent insertion without surgery.

According to the inclusion criteria and exclusion criteria, six patients without one-stage total resection of the primary site were excluded from the study. The other 78 patients were divided into a stent group (SG) or an emergency group (EG) according to their medical decision. Among the patients in SG, two patients failed to achieve clinical success and complicated with perforation. Thus, they underwent emergency surgery subsequently. They were moved into EG since they underwent emergency surgery, and the BTS procedure was not carried out, as has also been done in other studies. ${ }^{13,15}$ Thus, patients who underwent emergency surgery were placed in EG $(\mathrm{N}=41)$. Patients who accepted stent insertion as a BTS and underwent elective surgery were placed in $\mathrm{SG}(\mathrm{N}=37)$. The patient selection flowchart is shown in Figure 1.

\section{Procedure}

\section{Endoscopic stenting procedure}

In the Endoscopy Unit, stent placement was performed by two experienced endoscopists using a coloscope (CFH260AI, Olympus, Tokyo, Japan) with fluoroscopic guidance. ${ }^{13}$ The first step was intestinal cleaning. As the patients were complicated with obstruction, intestinal cleaning had to be completed using a low-pressure enema. ${ }^{16}$ The second step was colonoscopy examination. After the endoscopist identified the site of the obstruction and the colon lesion, a guide wire (Boston Scientific Jagwire $^{\mathrm{TM}}$, Marlborough, MA, USA) was passed through the narrowed lumen under fluoroscopic guidance. Then, a catheter (GT-2-T, Cook Medical, Winston-Salem, NC, USA) was passed through along with the guide wire. The third step was using water-soluble contrast material to 


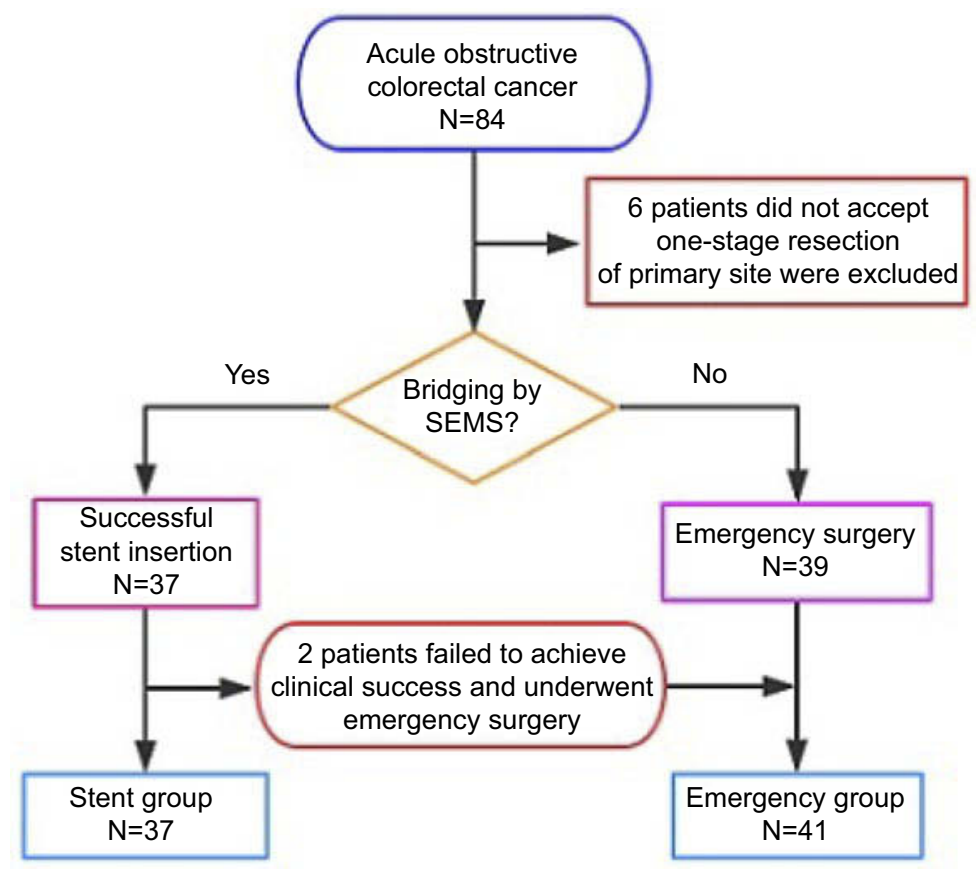

Figure I Patient selection flowchart. A total of 78 patients diagnosed with acute obstructive colorectal cancer who underwent elective surgery after stent insertion or emergency surgery from January 2013 to October 2016 were included in this study.

Abbreviation: SEMS, self-expanding metallic stent.

identify the lesion. After the contrast material was injected through the catheter, endoscopists could identify the tumor size, the length of lesion, the degree of stenosis and whether it was complicated with perforation. Fourth, a suitable self-expanding metallic stent (Micro-Tech (Nanjing), Nanjing, China) was chosen according to the length and caliber of the stricture (diameter, $20 \mathrm{~mm}$; length, $60-120 \mathrm{~mm}$ ). The stent was placed at least $2 \mathrm{~cm}$ over each side of the tumor. ${ }^{16}$ Normally, uncovered stents are used in our hospital and only one stent per procedure. More than one stent might be used when the lesion is too long, but the number of stents should no more than three. Finally, the proper position and expansion of the stent were assessed using fluoroscopic visualization. ${ }^{13}$ Technical success was defined as a stent in place when leaving the Endoscopy Unit and clinical success was defined as defecation within 72 -h post-procedure. ${ }^{4}$ The sites of the colonic obstructions are shown in Table S1.

\section{Surgical procedure}

Emergency surgery and stent insertion followed by one-stage laparoscopic or open surgery were based on the traditional colorectal cancer surgery methods. The surgical procedures in this study included two modalities: 1) hemicolectomy or greater (but less than total), right or left colectomy, where hemicolectomy is the removal of the total right or left colon and a portion of the transverse colon; 2) partial colectomy (less than hemicolectomy), such as enterocolectomy, ileocolectomy, cecectomy, partial resection of transverse colon and flexures and sigmoidectomy.

In this study, the definition of right and left-sided colon lesion was in accord with a previous study. ${ }^{17}$ Right-sided colon cancer was defined as the location of the tumor, including the cecum/appendix, ascending colon, hepatic flexure, and proximal transverse colon (proximal two-thirds of the transverse colon). Left-side colon cancer was defined as the location of the tumor, including the distal transverse colon (distal one-third of the transverse colon), splenic flexure, descending colon, and sigmoid colon. ${ }^{17}$ In addition, the tumor located at rectosigmoid colon and rectum were not included in this study.

\section{Follow-up data}

In the Colorectal Surgery Department, patients were followed initially at 3-month intervals for 2 years, every 6 months for the next 3 years, and then follow-up was performed once a year. If a patient did not return for observation after 1 year, information was obtained via a letter or telephone. All the patients were followed until death or until the end of the study in July 2018. Thus, all the patients were followed at least 20 months or until death. 
The quality of life was evaluated via the Quality of Life Questionnaire-Core 30 (QLQ-C30). QLQ-C30 is a questionnaire released by the European Organization for Research and Treatment of Cancer (EORTC) and is widely used in European countries and other countries around the world. In this study, six dimensions of the questionnaire were used to evaluate the quality of life of the survival patient in SG and EG, including global quality of life (GQL) and five functional dimensions such as physical function, role function, emotional function, cognitive function, and social function. The QLQ-C30 was used at the end of the study in July 2018, and the questionnaire surveys were carried out among survival patients. Twenty-three patients in SG (13 male and 10 female) and 21 patients in EG (12 male and nine female) were surveyed ( $P=\mathrm{NS}$, data not shown) by letter, telephone or return for observation.

\section{Statistical analysis}

Statistical analysis was performed using the statistical software package SPSS 22.0 (IBM Corp, Armonk, NY, USA). The Kaplan-Meier method was conducted to calculate the overall survival, and log-rank test was used to compare the survival difference between subdivisions. Univariate analyses were performed using the Mann-Whitney U analysis, Pearson's chi-square test, and Fisher's exact test. In addition, continuous variables were compared using the Student's $t$-test. $P$-values of less than 0.05 were considered significant.

\section{Results}

\section{Outcomes of stent insertion}

Of the 39 patients who accepted stent insertion for emergent decompression, all achieved technical success and left the Endoscopy Unit. Among these patients, 37 patients (94.9\%) achieved clinical success as defecation within $72 \mathrm{~h}$. Two patients failed to achieve clinical success and complicated with perforation. These two patients were subsequently treated with emergency surgery and were moved into EG. Patients who achieved clinical success were subsequently treated with nutrition support. The interval time between elective surgery and stent insertion was used to perform medical resuscitation, optimization of comorbid disorders, bowel preparation, and preoperative tumor staging. ${ }^{4,9}$ The median interval between stent insertion and elective surgery was 9 days (range 2-19). Complications were observed including perforation $(5.1 \%)$, obstruction $(7.7 \%)$ and migration
(2.6\%). The perforation was due to predilation to obtain access and excessive manipulation of the wire. ${ }^{18}$ Three patients in SG complicated with re-obstruction and one patient complicated with migration. The migration was attributed to dilatation prior to stent insertion, but the migration was only slight, and the patient was treated with an elective operation. Three patients were complicated with re-obstruction due to fecal impaction. The symptoms of two patients were relieved after a lowpressure enema intestinal cleaning. The symptoms of one patient were not relieved completely after intestinal cleaning, and this patient accepted elective surgery 2 days after stent insertion.

\section{Patient characteristics}

Patients were divided into two groups: $\mathrm{SG}(\mathrm{N}=37)$ and $\mathrm{EG}$ $(\mathrm{N}=41)$. The mean ages of $\mathrm{SG}$ and EG were $66.1 \pm 10.3$ (21 male and 16 female) and $65.4 \pm 11.7$ (25 male and 16 female), respectively. Both groups presented the same larger proportion which were observed in older age group (aged 60-79), abnormal carcinoembryogenic antigen (CEA) level before surgery ( $\geq 5 \mathrm{ng} / \mathrm{ml}$ ), left-sided colon cancer, pT3, pN0, pM0, AJCC stage II and III, moderately differentiated and adenocarcinoma. The proportion of patients who had an underlying disease was not significantly different between the two groups. In general, all the baseline characteristics were not significantly different between the two groups. The baseline characteristics of SG and EG are shown in Table 1.

\section{Comparison of preoperative condition and postoperative outcomes}

The preoperative general condition of patients in both groups was evaluated by serum albumin level and the American Society of Anesthesiologists Physical Status Classification System (ASA) risk score before surgery. The preoperative condition of SG was observed after BTS period and evaluated within $24 \mathrm{~h}$ before the elective surgery. The preoperative condition of EG was observed right before the emergency operation. The median serum albumin level of SG was 36.6 compared with 34.1 of EG $(P=0.020)$ (Figure 2$)$. The ASA risk score of SG was better than that of EG $(P=0.025)$ (Table 2).

The median operation time was $115 \mathrm{~min}$ and $180 \mathrm{~min}$ for SG and EG, respectively $(P<0.001)$. The median number of blood loss and exhausted time were the same in both groups, 
Table I Baseline characteristics of patients in SG and EG

\begin{tabular}{|c|c|c|c|c|}
\hline \multicolumn{2}{|l|}{ Characteristics } & \multirow{2}{*}{$\begin{array}{l}\mathbf{S G}(\mathbf{N}=\mathbf{3 7}) \\
21(56.8)\end{array}$} & \multirow{2}{*}{$\begin{array}{l}\text { EG }(\mathbf{N}=4 I) \\
25(6 I .0)\end{array}$} & \multirow{2}{*}{$\frac{\text { P-value }}{0.44 I}$} \\
\hline Gender, n (\%) & Male & & & \\
\hline & Female & $16(43.2)$ & $16(39.0)$ & \\
\hline \multirow[t]{3}{*}{ Age group, n (\%) } & $40-59$ & $13(35.1)$ & II (26.8) & 0.695 \\
\hline & $60-79$ & $20(54.1)$ & $24(58.5)$ & \\
\hline & $\geq 80$ & $4(10.8)$ & $6(14.6)$ & \\
\hline \multirow[t]{2}{*}{ Age (years,range) ${ }^{a}$} & & $66.1 \pm 10.3$ & $65.4 \pm 11.7$ & 0.790 \\
\hline & & $(45 \sim 84)$ & $(40 \sim 86)$ & \\
\hline \multirow[t]{2}{*}{ CEA before surgery, $\mathrm{n}(\%)$} & $<5 \mathrm{ng} / \mathrm{mL}$ & $16(43.2)$ & $19(46.3)$ & 0.482 \\
\hline & $\geq 5 \mathrm{ng} / \mathrm{mL}$ & $21(56.8)$ & $22(53.7)$ & \\
\hline \multirow[t]{2}{*}{ Tumor location, n (\%) } & Right-sided colon & II (29.7) & $15(36.6)$ & 0.345 \\
\hline & Left-sided colon & $26(70.3)$ & $26(63.4)$ & \\
\hline Underlying disease, n (\%) & & $16(43.2)$ & $19(46.3)$ & 0.482 \\
\hline \multirow[t]{3}{*}{ T stage, n (\%) } & $\mathrm{pT} 2$ & $6(16.2)$ & $5(12.2)$ & 0.877 \\
\hline & $\mathrm{pT} 3$ & $26(70.3)$ & $30(73.2)$ & \\
\hline & $\mathrm{pT} 4$ & $5(13.5)$ & $6(14.6)$ & \\
\hline \multirow[t]{3}{*}{$\mathrm{N}$ stage, $\mathrm{n}(\%)$} & $\mathrm{pNO}$ & $16(43.2)$ & $19(46.3)$ & 0.768 \\
\hline & $\mathrm{pNI}$ & $13(35.1)$ & $16(39.0)$ & \\
\hline & $\mathrm{pN} 2$ & $8(21.7)$ & $6(14.6)$ & \\
\hline \multirow[t]{2}{*}{ M stage, n (\%) } & pMO & $30(8 I . I)$ & $36(87.8)$ & 0.306 \\
\hline & pMI & $7(18.9)$ & $5(12.2)$ & \\
\hline \multirow[t]{3}{*}{ AJCC TNM stage , n (\%) } & II & $14(37.9)$ & $17(4 \mid .5)$ & 0.713 \\
\hline & III & $16(43.2)$ & $19(46.3)$ & \\
\hline & IV & $7(18.9)$ & $5(12.2)$ & \\
\hline \multirow[t]{3}{*}{ Histological grade, n(\%) } & Well-differentiated & $7(18.9)$ & II (26.8) & 0.710 \\
\hline & Moderately-differentiated & $23(62.2)$ & $23(56.1)$ & \\
\hline & Poorly-differentiated & $7(18.9)$ & $7(17.1)$ & \\
\hline \multirow[t]{2}{*}{ Histological type, n (\%) } & Adenocarcinoma & $33(89.2)$ & $37(90.2)$ & 0.548 \\
\hline & Mucous/signet-ring cell & $4(10.8)$ & $4(9.8)$ & \\
\hline
\end{tabular}

Note: ${ }^{a}$ Mean $\pm S D$.

Abbreviations: AJCC, American Joint Committee on Cancer; SG, stent group; EG, emergency group.

while the patients in SG could resume diet earlier $(P=0.015)$ and harvest more lymph nodes $(P=0.003)$. However, there was no significant difference in the postoperative hospital stay. In

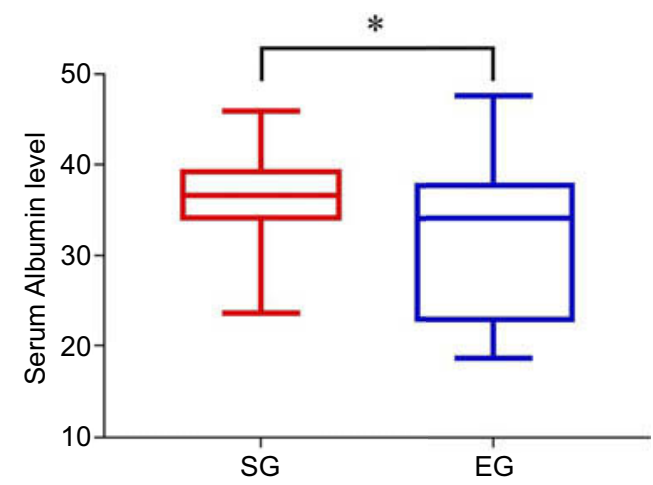

Figure 2 Analysis of preoperative serum albumin level of patients in SG and EG. The median of serum albumin level was 36.6 and 34.1 for the stent group and emergency group, respectively. * Statistically significant $(P<0.05)$.

Abbreviations: SG, stent group; EG, emergency group. addition, $78.4 \%$ of the patients in SG could examine more than 12 lymph nodes while only $51.2 \%$ of the patients in EG could examine enough lymph nodes according to the National Comprehensive Cancer Network (NCCN) guideline regarding lymph node examination. All patients in EG underwent open

Table 2 Preoperative condition of patients in SG and EG

\begin{tabular}{|c|c|c|c|c|}
\hline & & $\begin{array}{l}\text { SG } \\
(N=37)\end{array}$ & $\begin{array}{l}E G \\
(N=4 I)\end{array}$ & $P$-value \\
\hline $\begin{array}{l}\text { Serum albumin } \\
\text { level (g/L, range) } \\
\text { ASA risk score, } \\
\mathrm{n}(\%)\end{array}$ & $\begin{array}{l}\text { I/II } \\
\text { III/ } \\
\text { IV }\end{array}$ & $\begin{array}{l}36.6 \\
(23.7 \sim 45.9) \\
27(73.0) \\
10(27.0)\end{array}$ & $\begin{array}{l}34.1 \\
(18.7 \sim 47.6) \\
20(48.8) \\
21(51.2)\end{array}$ & $\begin{array}{l}0.020 \\
0.025\end{array}$ \\
\hline
\end{tabular}

Note: ${ }^{\text {Median. }}$

Abbreviations: SG, stent group; EG, emergency group; ASA, American Society of Anesthesiologists physical status classification system. 
surgery while 32 patients in SG underwent open surgery and the other five patients underwent laparoscopic surgery $(P=0.021)$. Total stoma creation was eight in $\mathrm{SG}$ versus 20 in EG $(P=0.011)$. In addition, 11 patients of EG suffered postoperative complications while six patients of SG suffered postoperative complications. In addition, no patients in $\mathrm{SG}$ died during hospitalization while two patients in EG died during hospitalization. The death was attributed to anastomotic leak and atelectasis which finally progressed to septic shock. However, the statistical result showed that there was no significant difference in complications and mortality between SG and EG. The results of postoperative outcomes are shown in Table 3.

\section{Long-term results of stent group and emergency group}

The long-term results consisted of survival benefit and quality of life. The median overall survival was 36 months for both groups. In addition, the overall survival was not significantly different between $\mathrm{SG}$ and EG $(P=0.909)$ (Figure 3). The Quality of Life Questionnaire-Core 30 (QLQ-C30) was used to evaluate the quality of life of the surviving patients in both groups. The standard scores

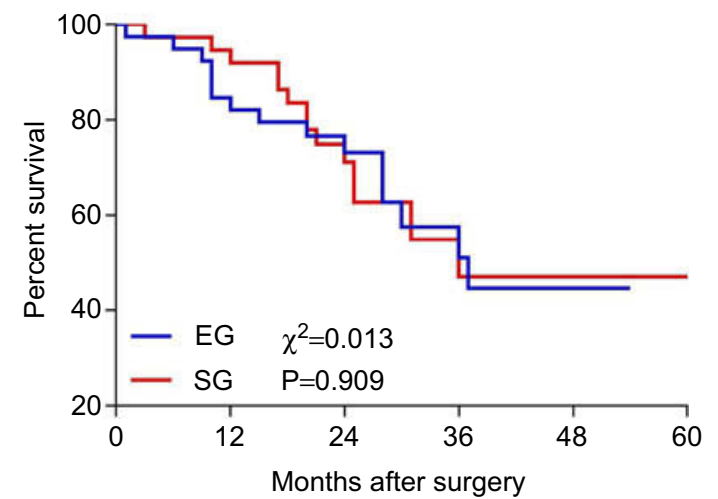

Figure 3 Overall survival of patients in SG and EG. The median overall survival was 36 months for both groups.

Abbreviations: SG, stent group; EG, emergency group.

were significantly different between SG and EG in five functional dimensions, and the GQL of patients in SG were better than that of EG $(P=0.026)$ (Table 4$)$.

\section{Discussion}

Ever since the first colonic stent placement was initially reported, considerable experience has been gathered regarding the clinical effectiveness in relieving obstruction with a median decompression rate of 94\%. ${ }^{19}$ However, consideration of the advantages and disadvantages of the

Table 3 Postoperative outcomes of patients in SG and EG

\begin{tabular}{|c|c|c|c|c|}
\hline & & SG $(\mathbf{N}=37)$ & EG $(N=4 I)$ & $P$-value \\
\hline Operation time (min,range) ${ }^{\mathrm{a}}$ & & $115(90-240)$ & $180(90-255)$ & $<0.001$ \\
\hline Blood loss (mL,range $)^{\mathrm{a}}$ & & $200(50-350)$ & $200(50-650)$ & 0.183 \\
\hline Exhaust time (day, range) ${ }^{\mathrm{a}}$ & & $3(I-5)$ & $3(1-6)$ & 0.105 \\
\hline Diet time (day, range $)^{a}$ & & $4(2-6)$ & $5(3-10)$ & 0.015 \\
\hline Postoperative hospital stay & & $11(6-31)$ & $12(6-40)$ & 0.105 \\
\hline \multicolumn{5}{|l|}{$(\text { day,range })^{\mathrm{a}}$} \\
\hline Harvested lymph nodes (range) ${ }^{a}$ & & $14(4-36)$ & II (4-26) & 0.003 \\
\hline \multirow[t]{2}{*}{ Harvested lymph nodes, n (\%) } & $<12$ & $8(21.6)$ & $20(48.8)$ & 0.011 \\
\hline & $\geq 12$ & $29(78.4)$ & $21(51.2)$ & \\
\hline \multirow[t]{2}{*}{ Surgery technique, $\mathrm{n}(\%)$} & Open & $32(86.5)$ & $41(100)$ & 0.021 \\
\hline & Laparoscopic & $5(13.5)$ & 0 & \\
\hline \multirow[t]{2}{*}{ Stoma, n (\%) } & Temporary & $3(8.1)$ & $6(14.6)$ & 0.295 \\
\hline & Permanent & $5(13.5)$ & $14(34.1)$ & 0.030 \\
\hline Total stoma creation, $\mathrm{n}(\%)$ & & $8(21.6)$ & $20(48.8)$ & 0.011 \\
\hline Postoperative complication, n (\%) & & $6(16.2)$ & II (26.8) & 0.196 \\
\hline Anastomotic leak, n (\%) & & $3(8.1)$ & $2(4.9)$ & $0.45 I$ \\
\hline Postoperative ileus, n (\%) & & $2(5.4)$ & $3(7.3)$ & 0.549 \\
\hline Atelectasis, $\mathrm{n}(\%)$ & & 0 & I (2.4) & 0.526 \\
\hline Wound abscess, n (\%) & & I (2.7) & $2(5.1)$ & 0.539 \\
\hline Intra-abdominal abscess, n (\%) & & 0 & $3(7.3)$ & 0.140 \\
\hline Mortality, n (\%) & & 0 & $2(4.9)$ & 0.273 \\
\hline
\end{tabular}

Note: ${ }^{\text {MMedian. }}$

Abbreviations: SG, stent group; EG, emergency group. 
Table 4 EORCT QLQ-C30 results of survival patients in SG and EG

\begin{tabular}{|l|l|l|l|}
\hline & SG (n=23) & EG (n=2 I) & P-value \\
\hline GQL $^{\text {a }}$ & 66.67 & 50.00 & 0.026 \\
Physical function $^{\text {a }}$ & 73.33 & 53.33 & 0.048 \\
Role function $^{\text {a }}$ & 50.00 & 33.33 & 0.023 \\
Emotional function $^{\text {a }}$ & 91.67 & 83.33 & 0.031 \\
Cognitive function $^{\text {a }}$ & 100.00 & 83.33 & 0.038 \\
Social function $^{\text {a }}$ & 50.00 & 33.33 & 0.025 \\
\hline
\end{tabular}

Note: ${ }^{\text {Median. }}$

Abbreviations: SG, stent group; EG, emergency group; GQL, global quality of life; EORTC, European Organization for Research and Treatment of Cancer; QLQ-C30, Quality of Life Questionnaire-Core 30.

SEMS and controversy regarding the survival benefit have not been defined yet.

In the early years, SEMS was used as an alternative option for palliative treatment. Several studies found that SEMS could present a palliative treatment option for incurable obstructive colon cancer. ${ }^{4,18,20}$ For patients in stage IV, distant metastasis might limit the possibility of radical resection while surgical decompression was required when complicated with obstruction. Researchers found that SEMS could decompress the large bowel obstruction instead of palliative colostomy for incurable colon cancer. ${ }^{18,20}$ Compared with palliative surgery, SEMS could reduce trauma and cost. In addition, stent placement might also reduce early morbidity, hospital stay and stoma creation in patients. ${ }^{20}$ However, survival benefits were not found in SG compared with the surgery group, and the surgery group even gained more survival benefit. ${ }^{18,20}$ In addition, several complications including migration, obstruction, perforation, and tenesmus might also contribute to the limitation of stenting application in incurable disease. ${ }^{18}$

Recently, SEMS has been used as a BTS to allow a single-stage surgical procedure later for resectable colon cancer associated with obstruction. SEMS gives the physicians the opportunities to complete preoperative examination and tumor staging, improve general condition, and perform bowel preparation. The advantage of SEMS as a BTS was that it could avoid colostomy as much as possible and allow for laparoscopic surgery and adequate lymphadenectomy, whereas its disadvantage laid in the increased perineural invasion (PNI) and decreased Onodera's prognostic nutritional index (OPNI) levels. ${ }^{13}$ Despite these disadvantages, SEMS as a BTS has been widely used globally in recent years. Therefore, a comparison of short-term and long-term outcomes between SEMS as a BTS and emergency surgery has aroused concern.
Several studies have found that short-term outcomes of the BTS group were better than that of EG. ${ }^{15,21-23}$ SEMS as a BTS could increase the chance of primary anastomosis and reduce the need for stoma creation and present better preoperative ASA risk score compared with emergency surgery. ${ }^{21,22}$ However, whether the BTS group had better results of post-procedural complications and mortality during hospitalization remains controversial. ${ }^{21,22}$ As for the long-term survival benefit, no significant difference was found between stent as a BTS and emergency surgery. ${ }^{15,23}$ These studies were carried out in patients with acute left-sided obstructive colorectal cancer, who represented a large proportion of patients. Patients suffering from acute right-sided obstructive colorectal cancer did exist, but they were not included in these studies. Thus, for all the patients with acute obstructive colorectal cancer, the short-term and long-term outcomes needed to be compared between SG and EG and the evaluation of the quality of life was still lacking.

In this study, 78 patients presented acute obstructive colorectal cancer and treated by one-stage resection of the primary site were finally included. There was no significant difference in the baseline characteristics between the two groups, which allowed for further study. The preoperative condition was better in SG, which might benefit from the adequate decompression, the recovery of general condition and nutrition support. A stent as a BTS could shorten the operation time and allow for laparoscopic surgery and adequate lymphadenectomy. In addition, the proportion of total stoma creation and permanent stoma construction in SG was decreased. These advantages were in accord with previous studies. ${ }^{13,21,22}$ The better general condition after stent insertion, more sufficient decompression, and better large bowel preparation before elective surgery might ensure successful laparoscopic operation and primary anastomosis without increasing the rate of anastomotic leak.

Stent-associated complications were observed including perforation, obstruction, and migration. The perforations were due to predilation to obtain access, and excessive manipulation of the wire. ${ }^{18}$ The patients complicated with perforation underwent emergency surgery to resect the tumor and the perforation site. Fortunately, no serious complications and death were caused by the perforation during hospitalization. The migration was attributed to dilatation prior to stent insertion but was only slight and the patient was treated by elective surgery. Three patients were complicated with re-obstruction due 
to fecal impaction. The symptoms of two patients were relieved after a low-pressure enema intestinal cleaning. The symptoms of one patient were not relieved completely after intestinal cleaning, and this patient accepted elective surgery 2 days after stent insertion. These were common complications of stent procedure, and patients were not seriously affected.

The mortality and complications of surgery in SG were less than in EG. However, the hospital stay, complications and mortality were not significantly different according to statistical analysis and the results were similar to another study. ${ }^{15}$ A possible explanation might be that those variables were significantly affected by individual conditions including age, gender, and many other factors. Several studies found that SEMS as a BTS might be more dangerous because of poor long-term oncologic outcomes, such as short overall survival. $^{24,25}$ However, we found that the overall survival was not significantly different between two groups and the results were in accord with other studies. ${ }^{21,23}$ In addition, the quality of life was better in SG. The patients in SG might benefit from the decrease of stoma creation and the increase of a single-stage procedure which helped them to return to normal life more easily and avoid re-operation compared with patients in EG.

The researchers acknowledged several study limitations. In this retrospective study, selection biases could not be avoided. In addition, SEMS was not used as a routine measure in our hospital until the past few years and this might contribute to the limitation of the cases and the follow-up time. In addition, inaccurate responses to questionnaires from the surviving patients might also contribute to the study limitations.

Despite these limitations, this study enrolled both left-sided and right-sided colon cancer patients with acute colonic obstruction and comprehensively studied the baseline characteristics, preoperative condition, postoperative outcomes, long-term survival outcomes and the quality of life to explore the SEMS as a safe and feasible alternative option for emergency surgery in the management of acute obstructive colorectal cancer.

\section{Conclusion}

In this study, we found that there was no significant difference in the baseline characteristics between the two groups. The preoperative condition, operation time, diet time and harvested lymph nodes were better in SG compared with those in EG. In addition, the proportion of total stoma creation and permanent stoma creation was decreased and patients who underwent laparoscopic surgery were increased in SG. The hospital stay, complications and mortality were not significantly different between the two groups. In addition, the overall survival was not significantly different while the quality of life of SG was better than that of EG. Thus, the stent as a BTS seems to be a safe and feasible alternative option for emergency surgery in the management of acute obstructive colorectal cancer.

\section{Ethics approval and consent to participate}

This retrospective study was approved by the Institutional Review Board of the Second Affiliated Hospital of Harbin Medical University. All methods were carried out in accordance with the Declaration of Helsinki and the approved guidelines. All participants provided their written informed consent.

\section{Abbreviations list}

SEMS, self-expanding metallic stent; BTS, bridge to surgery; ASA, American Society of Anesthesiologists physical status classification system; CRC, colorectal cancer; CT, computed tomography; MDT, multidisciplinary treatment; SG, stent group; EG, emergency group; GQL, global quality of life; EORTC, European Organization for Research and Treatment of Cancer; QLQ-C30, Quality of Life Questionnaire-Core 30; CEA, carcinoembryogenic antigen; NCCN, National Comprehensive Cancer Network; PNI, perineural invasion; OPNI, Onodera's prognostic nutritional index. AJCC, American Joint Committee on Cancer.

\section{Availability of data and materials}

The datasets used and/or analyzed during the current study are available from the corresponding author on reasonable request.

\section{Acknowledgment}

This project was funded by China Postdoctoral Science Foundation (Grant No. 2018M641856) and Hei Long Jiang Postdoctoral Foundation.

\section{Author contributions}

YLMW, GYW: Substantial contributions to project conceptualization, data curation, and formal analyses, data presentation/ visualization, interpretation of data, drafting of the original manuscript, revising and editing final manuscript. $\mathrm{HQH}$ and MW: Substantial contributions to interpretation of data, drafting of the original manuscript, revising and editing final manuscript. XH and QZ: Substantial contributions to interpretation 
of data, data curation, and formal analyses, revising and editing final manuscript. LY: Substantial contributions to interpretation of data, data presentation/visualization, drafting of the original manuscript, revising and editing final manuscript. YGC: Substantial contributions to data presentation/visualization, revising and editing final manuscript. All authors gave approval for the final version to be published and agreed to be accountable for all aspects of the work.

\section{Disclosure}

The authors report no conflicts of interest in this work.

\section{References}

1. Siegel RL, Miller KD, Jemal A. Cancer statistics, 2018. CA Cancer J Clin. 2018;68(1):7-30. doi:10.3322/caac.21442

2. Pisano M, Zorcolo L, Merli C, et al. 2017 WSES guidelines on colon and rectal cancer emergencies: obstruction and perforation. World J Emerg Surg. 2018;13:36. doi:10.1186/s13017-018-0192-3

3. Cheynel N, Cortet M, Lepage C, Benoit L, Faivre J, Bouvier A-M. Trends in frequency and management of obstructing colorectal cancers in a well-defined population. Dis Colon Rectum. 2007;50 (10):1568-1575. doi:10.1007/s10350-007-9007-4

4. Angenete E, Asplund D, Bergström M, Park P-O. Stenting for colorectal cancer obstruction compared to surgery - a study of consecutive patients in a single institution. Int $J$ Colorectal Dis. 2012;27 (5):665-670. doi:10.1007/s00384-011-1374-6

5. Ascanelli S, Navarra G, Tonini G, et al. Early and late outcome after surgery for colorectal cancer: elective versus emergency surgery. Tumori. 2003;89(1):36-41.

6. Alvarez JA, Baldonedo RF, Bear IG, Truán N, Pire G, Alvarez P. Presentation, treatment, and multivariate analysis of risk factors for obstructive and perforative colorectal carcinoma. Am $J$ Surg. 2005;190(3):376-382. doi:10.1016/j.amjsurg.2005.01.045

7. Sjo O, Larsen S, Lunde O, Nesbakken A. Short term outcome after emergency and elective surgery for colon cancer. Colorectal Dis. 2008;11(7):733-739. doi:10.1111/j.1463-1318.2008.01613.x

8. Tilney HS, Lovegrove RE, Purkayastha S, et al. Comparison of colonic stenting and open surgery for malignant large bowel obstruction. Surg Endosc. 2007;21:225-233. doi:10.1007/s00464-005-0644-1

9. Martinez-Santos C, Lobato RF, Fradejas JM, et al. Self-expandable stent before elective surgery vs. emergency surgery for the treatment of malignant colorectal obstructions: comparison of primary anastomosis and morbidity rates. Dis Colon Rectum. 2002;45:401-406.

10. Khot UP, Lang AW, Murali K, Parker MC. Systematic review of the efficacy and safety of colorectal stents. Br J Surg. 2002;89:1096-1102. doi:10.1046/j.1365-2168.2002.02148.x

11. Watt AM, Faragher IG, Griffin TT, Rieger NA, Maddern GJ. Selfexpanding metallic stents for relieving malignant colorectal obstruction: a systematic review. Ann Surg. 2007;246:24-30. doi:10.1097/01. sla.0000261124.72687.72
12. Maruthachalam K, Lash GE, Shenton BK, Horgan AF. tumor cell dissemination following endoscopic stent insertion. $\mathrm{Br} J$ Surg. 2007;94:1151-1154. doi:10.1002/bjs.5790

13. Haraguchi N, Ikeda M, Miyake M, et al. Colonic stenting as a bridge to surgery for obstructive colorectal cancer: advantages and disadvantages. Surg Today. 2016;46(11):1310-1317. doi:10.1007/ s00595-016-1333-5

14. Yamashita S, Tanemura M, Sawada G, et al. Impact of endoscopic stent insertion on detection of viable circulating tumor cells from obstructive colorectal cancer. Oncol Lett. 2018;15(1):400-406. doi:10.3892/ol.2017.7339

15. Kim HJ, Huh JW, Kang WS, et al. Oncologic safety of stent as bridge to surgery compared to emergency radical surgery for left-sided colorectal cancer obstruction. Surg Endosc. 2013;27(9):3121-3128. doi:10.1007/s00464-013-2865-z

16. Li ZX, Wu XH, Wu HY, et al. Self-expandable metallic stent as a bridge to elective surgery versus emergency surgery for acute malignant colorectal obstruction. Int $J$ Colorectal Dis. 2016;31 (3):561-570. doi:10.1007/s00384-015-2476-3

17. Imperial R, Ahmed Z, Toor OM, et al. Comparative proteogenomic analysis of right-sided colon cancer, left-sided colon cancer and rectal cancer reveals distinct mutational profiles. Mol Cancer. 2018;17 (1):177. doi:10.1186/s12943-018-0923-9

18. Fernández-Esparrach G, Bordas JM, Giráldez MD, et al. Severe complications limit long-term clinical success of self-expanding metal stents in patients with obstructive colorectal cancer. $\mathrm{Am}$ J Gastroenterol. 2010;105(5):1087-1093. doi:10.1038/ajg.2009.660

19. Sebastian S, Johnston S, Geoghegan T. Pooled analysis of the efficacy and safety of self-expanding metal stenting in malignant colorectal obstruction. Am J Gastroenterol. 2004;99(10):2051-2057. doi:10.1111/j.1572-0241.2004.40017.x

20. Súarez J, Jiménez J, Vera R, et al. Stent or surgery for incurable obstructive colorectal cancer: an individualized decision. Int J Colorectal Dis. 2010;25:91-96. doi:10.1007/s00384-009-0814-z

21. Lee GJ, Kim HJ, Baek JH, Lee W-S, Kwon KA. Comparison of short-term outcomes after elective surgery following endoscopic stent insertion and emergency surgery for obstructive colorectal cancer. Int $J$ Surg. 2013;11(6):442-446. doi:10.1016/j. ijsu.2013.04.010

22. Zhang Y, Shi J, Shi B, Song C-Y, Xie W-F, Chen Y-X. Selfexpanding metallic stent as a bridge to surgery versus emergency surgery for obstructive colorectal cancer: a meta-analysis. Surg Endosc. 2012;26(1):110-119. doi:10.1007/s00464-011-1835-6

23. Kwak MS, Kim WS, Lee JM, et al. Does stenting as a bridge to surgery in left-sided colorectal cancer obstruction really worsen oncological outcomes? Dis Colon Rectum. 2016;59(8):725-732. doi:10.1097/DCR.0000000000000631

24. Sabbagh C, Browet F, Diouf M, et al. Is stenting as "a bridge to surgery" an oncologically safe strategy for the management of acute, left-sided, malignant, colonic obstruction? a comparative study with a propensity score analysis. Ann Surg. 2013;258:107-115. doi:10.1097/SLA.0b013e31827e30ce

25. Pirlet IA, Slim K, Kwiatkowski F, Michot F, Millat BL. Emergency preoperative stenting versus surgery for acute left-sided malignant colonic obstruction: a multicenter randomized controlled trial. Surg Endosc. 2011;25:1814-1821. doi:10.1007/ s00464-010-1471-6 


\section{Supplementary material}

Table SI The obstruction site of SG and EG

\begin{tabular}{|l|l|l|l|}
\hline Obstruction site & SG (n=37) & EG (n=4 I) & P-value \\
\hline Cecum & 3 & 3 & $0.87 \mid$ \\
Ascending colon & 5 & 7 & \\
Hepatic flexure & 2 & 4 & \\
Transverse colon & 4 & 3 & \\
Splenic flexure & 4 & 4 & \\
Descending colon & 6 & 3 & \\
Sigmoid colon & 13 & 17 & \\
\hline
\end{tabular}

Abbreviations: SG, stent group; EG, emergency group.

\section{Publish your work in this journal}

Cancer Management and Research is an international, peer-reviewed open access journal focusing on cancer research and the optimal use of preventative and integrated treatment interventions to achieve improved outcomes, enhanced survival and quality of life for the cancer patient.

The manuscript management system is completely online and includes a very quick and fair peer-review system, which is all easy to use. Visit http://www.dovepress.com/testimonials.php to read real quotes from published authors. 